\title{
Fecal Microbiota Transplantation in Patients with Primary Sclerosing Cholangitis: A Pilot Clinical Trial.
}

Authors: Jessica R. Allegretti ${ }^{1,2}$, Zain Kassam³, Madeline Carrellas ${ }^{1}$, Benjamin H. Mullish $^{4}$, Julian R. Marchesi ${ }^{4}$, Alexandros Pechlivanis ${ }^{4}$, Mark Smith ${ }^{3}$, Ylaine Gerardin ${ }^{3}$, Sonia Timberlake ${ }^{3}$, Daniel S. Pratt ${ }^{2,5}$, and Joshua R. Korzenik ${ }^{1,2}$

1. Division of Gastroenterology, Hepatology and Endoscopy, Brigham and Women's Hospital, Boston MA

2. Harvard Medical School, Boston, MA

3. Finch Therapeutics Group, Somerville, MA

4. Division of Integrative Systems Medicine and Digestive Disease, Faculty of Medicine, Imperial College London, London, United Kingdom

5. Division of Gastroenterology, Massachusetts General Hospital, Boston, MA

Keywords: Fecal Microbiota Transplantation, Microbiome, Inflammatory Bowel Disease, Stool Transplant, Alkaline Phosphatase

\section{Corresponding Author:}

Jessica R. Allegretti, MD, MPH

Brigham and Women's Hospital, Division of Gastroenterology

75 Francis Street, Boston, MA 02115, USA

$+1-617-732-6389$

jallegretti@bwh.harvard.edu

Conflicts of Interest: JRA consults for and has research support from Finch Therapeutics Group. YG, ST, MS and ZK are employees of Finch Therapeutics Group. 
Author Contributions: JRA initiated study concept and design acquisition of data, analysis and interpretation of data, and drafting of the manuscript. YG, ST, MS analyzed and interpreted microbiome sequencing results. BHM, AP and JRM analyzed and interpreted the metabolomics results. MC participated in data acquisition. ZK participated in interpretation of data and critical revision of the manuscript DP and JK provided critical revisions of manuscript.

Funding: The trial was funded by PSC Partners seeking a Cure. BHM is the recipient of a Medical Research Council Clinical Research Training Fellowship (grant reference: MR/R000875/1). The Division of Integrative Systems Medicine and Digestive Disease at Imperial College London receives financial support from the National Institute for Health Research (NIHR) Imperial Biomedical Research CenFuntre (BRC) based at Imperial College Healthcare NHS Trust and Imperial College London.

\section{STUDY HIGHLIGHTS:}

\section{WHAT IS CURRENT KNOWLEDGE}

- Primary Sclerosing Cholangitis (PSC) is a chronic cholestatic liver condition with no approved therapies

- The microbiome has been implemented in the pathogenesis of PSC

- FMT has been shown to restore the microbiome in other chronic disease.

\section{WHAT IS NEW HERE}

- FMT is safe in patients with PSC

- strong engrafter OTUs correlate with a decrease in Alkaline Phosphatase. 


\begin{abstract}
Background: Primary sclerosing cholangitis (PSC) is a cholestatic liver disease with no effective medical therapies. A perturbation of the gut microbiota has been described in association with PSC, and fecal microbiota transplantation (FMT) has been reported to restore the microbiome in other disease states. Accordingly, we aimed to evaluate the safety, change in liver enzymes, microbiota and metabolomic profiles in PSC patients after FMT.
\end{abstract}

Methods: Open-label pilot study of PSC patients with concurrent inflammatory bowel disease (IBD) and ALP $>1.5 \mathrm{X}$ the upper limit of normal. Participants underwent a single FMT by colonoscopy. Liver enzyme profiles and stool microbiome and metabolomic analysis was conducted at baseline and week 1, 4, 8, 12 and 24 post-FMT. The primary outcome was safety and secondary outcomes include a decrease in ALP $\geq 50 \%$ from baseline by week 24 post-FMT, as well as stool microbiota (by 16S rRNA gene profiling) and metabonomic dynamics were assessed.

Results. Ten patients underwent FMT. Nine patients had ulcerative colitis and 1 with Crohn's colitis. The mean baseline ALP was 489 U/L. There were no related adverse events. Overall, 30\% (3/10) experienced a $\geq 50 \%$ decrease ALP. The diversity increased in all patients post-FMT, as early as week $1(\mathrm{p}<0.01)$. Importantly, abundance of engrafter operational taxanomic units (OTUs) in patients post-FMT correlated with decreased ALP $(p=0.02)$.

Conclusion: To our knowledge, this first study to demonstrate that FMT in PSC is safe. Additionally, increases in bacterial diversity and engraftment may correlate with an improvement in ALP among PSC patients. 


\section{Introduction:}

Primary sclerosing cholangitis (PSC) is a progressive, chronic cholestatic liver disease characterized by inflammatory and fibrotic destruction of the intrahepatic and/or extrahepatic bile ducts. PSC often progress to biliary cirrhosis, portal hypertension and liver failure $(1,2)$. In up to $80 \%$ of patients $(3)$, ulcerative colitis (UC) or Crohn's disease (CD) will also be present (1). Medications used for the treatment of UC have not been effective in reducing inflammation or bringing about remission in PSC $(4,5)$. Currently, there are no Food and Drug Administration (FDA) approved medical therapy for PSC.

It has been postulated that bacteria may stimulate an aberrant immune response resulting in the perpetuation of the biliary inflammation seen in PSC. One hypothesis is that bacteria gain access to the liver and biliary tree through translocation across an abnormal and inflamed intestinal mucosa into the portal venous system $(5,6)$. Additionally, the immune reaction in PSC is thought to be mediated by autoantibodies, including pANCA, that recognize the bacterial antigen cell division protein FtsZ (7). Animal models have demonstrated that an enteric dysbiosis can lead to hepatobiliary inflammation with features similar to PSC (5). A dysbiosis has been identified in patients with PSC, distinct from those with IBD without PSC. Additionally, unique microbial signatures have been identified in the bile of patients with PSC, strengthening this hypothesis $(8,9)$. However, the concept of a general dysbiosis is controversial and in this paper we will use it as a dependent change in the hosts' microbiota, which falls outside of the normal parameter space for that individual (10) .

However, there has been limited success using antibiotics in treating PSC. Metronidazole has been shown to result in modest improvement in liver enzymes (11). Oral vancomycin has also been advanced as a potentially promising therapy (12). An initial report of three pediatric patients and a subsequent small, uncontrolled series of oral vancomycin in 14 children showed improvement in liver tests and symptoms $(13,14)$; however, this has not been replicated in adults (15) . 
Recent advances in fecal microbiota transplantation (FMT) are changing the treatment paradigm for recurrent Clostridioides difficile infection, but are also leading to a better understanding of the microbial contribution to other chronic diseases (16). Four randomized trials and numerous case reports suggest FMT may be a promising therapy for UC (17). Therefore, restoration of the microbiome is a reasonable target for therapy in PSC, as there is no treatment, given the complexity of PSC-IBD and the known interactions between genes, mucosal immunity, immune cell tracking, and the microbiome (18).

\section{Methods:}

\section{$\underline{\text { Patient Selection }}$}

An open-label investigator-initiated pilot, single center academic study (Brigham and Women's Hospital Clinical Trials Center) enrolled PSC patients with concurrent inflammatory bowel disease (IBD) between January 2016 and June 2017 (19). Eligible patients were adults age 18 or greater and required a confirmed diagnosis of PSC with typical cholangiographic findings $>6$ months in duration, ALP $>1.5 \mathrm{X}$ the upper limit of normal and no evidence of cirrhosis on last magnetic resonance cholangiopancreatography (within 6 months). In addition, patients were required to have documentation of typical histopathology for UC or CD with colonic involvement. A 4-week washout period for ursodeoxycholic acid (UDCA) was required. Exclusion criteria included cirrhosis, isolated small bowel CD, patients who were pregnant or nursing, patients who were unable or unwilling to undergo a colonoscopy, active malignancy, chronic kidney disease (GFR $<60 \mathrm{~mL} / \mathrm{min} / 1.72 \mathrm{~m} 2$ ), or history of valvular heart disease. Patients who had taken UDCA within 4 weeks of screening, antibiotics within 2 months of screening, or probiotics within 1 month of screening were excluded. Patients on biologic therapy for their IBD were also excluded. Azathioprine and mesalamine/5-ASA were permitted as concomitant therapies.

Liver enzyme profiles, stool microbiota analysis and metabolomic profiling was conducted at baseline and week 1, 4, 8, 12 and 24 post-FMT. The primary outcome was safety. The secondary efficacy outcome was a decrease in ALP $\geq 50 \%$ from baseline at any point during the 24-week post-FMT follow up period. ALP was selected to be aligned with international consensus guidelines (20), though a more conservative target of $\geq 50 \%$ decrease was chosen 
given its imprecision. Additional secondary outcomes include microbial diversity postFMT when compared to baseline as well as bile acid profiles post FMT compared to preFMT samples and the donor. The study protocol conformed to the ethical guidelines of the 1975 Declaration of Helsinki and was approved by the institutional review board at the Brigham and Women's Hospital and all patients provided written informed consent prior to participation (NCT02424175). Additionally, FDA approval via investigational new drug application (IND 16324, 2015 ) was obtained.

Given there is no data on the effect size of FMT in PSC, this was designed as a proof of concept safety study. A sample size of 10 was chosen for feasibility given the rarity of this disease.

\section{Fecal Microbiota Transplantation Preparation:}

Screening of the Donor: Donor material was produced at a large stool bank based on a previously described protocol (OpenBiome, Somerville) (21). Briefly, donors were subjected to rigorous health and infection screening processes. Potential donors underwent an on-site clinical assessment and a 200-point health questionnaire. Thereafter, the potential donor undergoes a battery of stool and serological tests, aimed at screening for bacterial, viral and fungal infectious diseases (22).

\section{Fecal Microbiota Transplantation}

All participants underwent a standard of care bowel prep with polyethylene glycol on the day before the colonoscopy. No antibiotics were administered prior to the FMT. Colonoscopy was performed to the cecum with fecal material administered in the right colon. All subjects received material $(90 \mathrm{~mL})$ from a single donor.

\section{Microbiota Composition Analysis}

16S rRNA gene sequencing

Stool samples were collected for sequencing from donors and patients at baseline, 1, 4, 8, 12 and 24 weeks post-FMT. Samples were stored by flash freezing at $-80^{\circ} \mathrm{C}$. DNA 
extraction, PCR amplification of the 16S rRNA gene's V4 region, and Illumina paired-end sequencing was performed at the University of Michigan core facility, as described previously (23).

\section{S rRNA gene processing}

Primers were trimmed, paired ends merged, and de novo operational taxonomic units (OTUs) identified with a custom pipeline. In order to have maximum resolution for engraftment analysis, OTUs were defined by unique $16 \mathrm{~S}$ rRNA gene sequences, i.e. 100\% sequence identity. OTUs represented in fewer than two unique samples were discarded, resulting in a final median depth of 26,988 reads/sample and 64,510 OTUs across the study. Taxonomic assignments for each OTU were called using UTAX trained on the RDP database.

\section{$\underline{\text { Bacterial community analysis }}$}

Samples were rarefied to the lowest sample read count (2592 reads) for alpha diversity analysis. Paired t-tests were used to compare diversity or donor similarity before vs. after FMT, and independent t-tests were used for all other comparisons. Because previous reports have consistently shown low diversity in PSC microbiomes $(24,25)$ and that healthy-donor FMT increases diversity (26), all reported p-values are one-sided. For each patient, engrafting OTUs were identified based on presence in both the FMT donor and the patient's first post-FMT stool sample, as well as depletion in the patient's pre-FMT stool sample. Engrafting OTUs were classified as 'frequent engrafters' if they were observed as engrafting in greater than half the patients. Correlations to ALP improvement were performed by transforming unrarefied OTU read counts to relative abundances, subtracting baseline abundances, averaging across all post-FMT samples for each patient, and calculating the Spearman rank correlation to the patient's baseline-normalized ALP level at 24 weeks post-FMT.

\section{Ultra-performance liquid chromatography-mass spectrometry (UPLC-MS) profiling and analysis of fecal bile acids:}


Stool sample preparation for UPLC_MS was performed as previously described (27). Stool extract bile acid analysis was obtained using ACQUITY UPLC (Waters Ltd, Elstree, UK) coupled to a Xevo G2 Q-ToF mass spectrometer equipped with an electrospray ionization source operating in negative ion mode, using the method described by Sarafian and colleagues (28).

Quality control (QC) samples were prepared through the pooling of equal volumes of the fecal filtrates. QC samples were used as an assay performance monitor (29), and as a proxy to remove features with high variation. In addition, QC samples were spiked with mixtures of bile acid standards (55 bile acid standards including 36 non-conjugated, 12 conjugated with taurine, seven conjugated with glycine (Steraloids, Newport, RI, USA)) and were coanalyzed with the stool extracts to determine the chromatographic retention times of bile acids and to facilitate identification of metabolites.

Waters raw data files were converted to NetCDF format and data were extracted using XCMS (v1.50) package with R (v3.1.1) software. Probabilistic quotient normalization (30) was applied to correct for dilution effects and chromatographic features with coefficient of variation $>30 \%$ in QC samples were excluded from further analysis. The relative intensities of the features were corrected to the dry weight of the stool samples. Secondary bile acids were defined as those produced from primary bile acids via gut microbial 7alpha-dehydroxylation, whilst tertiary bile acids were those produced from primary bile acids via processes involving other forms of microbial modification, e.g. 7alpha-/betaisomerization of chenodeoxycholic acid (CDCA) to form UDCA.

Multivariate analysis of UPLC-MS bile acid profiling data was performed on pareto-scaled data. Statistical analysis of bile acid data was performed using GraphPad Prism, v7.03.

\section{Results.}

Ten patients were enrolled and underwent a single FMT from a single donor. Among the cohort, the mean age was 41.1 years (SD 15.7), 8 (80\%) patients were male and $9(90 \%)$ had large duct PSC. Nine patients had concurrent UC (6 with total Mayo Score 0, 3 with 
total Mayo Score 1) and 1 with Crohn's colitis (Harvey Bradshaw Index 0 and Crohn's Disease Endoscopic Index of Severity 0). The mean baseline ALP was 489 U/L (Table 1). Six patients were withdrawn from UDCA prior to start of the study.

With regard to the primary outcome, safety, there were no related adverse events (AEs). Specifically, there were no serious and not serious related AEs including deaths or infectious complications. Only one serious adverse event was noted and felt to be unrelated to the FMT. One patient had an episode of sinusitis that occurred 54 days post-FMT and required an emergency room visit as well as a course of doxycycline. In addition, one patient did not tolerate being off UDCA and after discussion with his treating hepatologist this treatment was restarted prior to the week 4 visit. Given any changes in liver biochemistries would be difficult to interpret in this patient, we have removed this patient from the per protocol (PP) analysis.

With regards to the secondary analysis, overall, the Intention to Treat (ITT) analysis revealed 3/10 (30\%) patients experienced a $\geq 50 \%$ decrease ALP. Although less important in cholestatic disease, $70 \%(7 / 10)$ experienced a 30\% decrease (exploratory post-hoc analysis) in at least one of their serum liver enzymes (ALT, AST ) post-FMT.

In terms of the per protocol analysis (PP), 33\% (3/9) of PSC patients experienced a $\geq 50 \%$ decrease ALP (Figure 1) and 77\% (7/9) experienced a 30\% decrease in at least one of their liver biomarkers (ALT, AST) post-FMT.

\section{Microbiota Composition Analysis}

The composition of the microbiota of patients with PSC differed from that of the donor at baseline pre-FMT and is more similar to the composition of the donor 1-week post FMT (Figure 2, Figure 3). Additionally, there was a trend toward lower pre-FMT PSC community diversity compared to that of healthy donors, (Figure 3, $\mathrm{p}=0.053$ ). Diversity and similarity to donor increased in all patients post-FMT, with changes seen as early as week $1(p=<0.01)$ (Figure 3) and maintained an upward trend throughout week 24 (Figure 3). Most patients showed global microbiome engraftment signals, including 
increased diversity and similarity to donor, that were stable over the study duration (Figure 3). A total of 2204 engrafter OTUs, defined as OTUs present in the donor, missing pre-FMT and present in patients 1-week post FMT, were delivered (Figure 4). Engrafter OTUs represented a diverse set of taxonomic classes, and included Desulfovibrio and Faecalibacterium, which have been previously identified as depleted in PSC $(25,31)$. Importantly, abundance of frequently-engrafting OTUs in patients postFMT tended to be correlated with decreased ALP (Figure 5). OTUs associated with ALP improvement were enriched among the OTUs engrafting in greater than half the patients, including the short-chain fatty acid producing genera Odoribacter, Alistipes, and Erysipelotrichaceae insertae sedis (32).

\section{UPLC-MS bile acid profiling data:}

Multi-variate analysis of UPLC-MS bile acid profiling data by PCA demonstrated clustering of serial samples by study participant (Figure 6A), but there was no clear effect of FMT upon stool bile acid profile clustering (Figure 6B). No valid supervised multivariate models could be constructed between stool bile acid profiles pre-FMT and postFMT at any time point, consistent with FMT having no statistically significant effect upon bile acid profiles; however, bile acid profiles pre-FMT were similar in overall composition to that of the donor (Figure 6C).

Multi-variate regression analysis between ALP levels and all mean integrated peaks from spectra was performed using PLS, with excellent correlation identified $(p<0.0001)$. On Spearman's rank analysis across all samples, a negative correlation was found between liver enzymes and relative UDCA levels $(r=-0.423$ for ALP, $r=-0.53$ for AST, $r=-0.61$ for ALT) and percentage of tertiary bile acids of total bile acids ( $r=-0.40$ for ALP, $r=-$ 0.56 for AST, $r=-0.61$ for ALT). A positive correlation was found between liver enzymes and percent of amino-conjugate bile acids as a percentage of total bile acids $(r=0.39$ for ALP, $r=0.41$ for AST, $r=0.43$ for ALT). On linear regression analysis across all samples, a negative correlation was found between ALP and $\%$ tertiary bile acids ( $p=0.0028$ ) (Figure 7A), but a positive correlation between ALP and \% tauro-conjugates $(p=0.01)$ (Figure $7 \mathrm{~B}$ ) and $\%$ glyco-conjugates $(p=0.0004)$ (Figure $7 \mathrm{C})$. 


\section{Discussion}

To our knowledge, this is the first study to demonstrate that FMT is safe in PSC. Additionally we found that in PSC, FMT increases bacterial diversity, and that engraftment may correlate with an improvement in ALP among PSC patients. It has been demonstrated that the gut microbiome of patients with both PSC and IBD is unique to that of patients with only IBD as well as healthy controls, although the specific microbial signature are not consistent $(25,33)$. However, the role of the microbiome in the pathogenesis of this disease remains unclear.

This study demonstrated that FMT was able to increase bacterial diversity as early as week 1, with persistence through to week 24, indicating that a single FMT may be sufficient to induce engraftment in this population. However, it has been reported that multiple FMTs are required to obtain remission in UC (34). Accordingly, we speculate that it may be possible to observe a further decrease in the ALP if multi-FMT dosing in patients with PSC are performed. This emphasizes the need for dose-finding and dose regimen studies in this nascent field.

We report a correlation between the abundance of engrafter OTUs and a decrease in ALP levels. ALP levels improved in 33\% of patients, albeit with variable dynamics, after FMT. When looking at overall alpha diversity this correlation was not appreciated, although alpha diversity is an over simplified metric. However, when you evaluate individual, frequently-engrafting OTUs, ALP improvement was appreciated. In particular these frequently engrafting OTUs included genera capable of producing short-chain fatty acids, which are known to be depleted In IBD (35).

Overall bile acid profiles did not change after FMT. This raises the question of whether FMT acts through alterations in gut microbiota-metabolomic interactions involving metabolites other than bile acids. Our multi-variate regression analysis (PLS) showed excellent correlation between ALP levels and spectral features which does contain some lipid classes. Since we did not use authentic standards for those, it is difficult to formally identify these by this technique alone; however, such metabolites are likely to be an 
additional explanation for the regression results. As such, it may be hypothesized that FMT may be improving hepatic function, underscored by improved ALP, in PSC patients through a downstream effect upon key lipid metabolites. So even though no changes in bile acids were observed, FMT still may be having a positive functional effect mediated through the immune system (36).

This study has several limitations. First, it was an uncontrolled, small pilot trial and the secondary clinical outcome assessments was not blinded. However, a strong placebo effect seems unlikely in patients with a chronic, progressive disease with an objective lab-based outcome. The merits of ALP as a marker of disease are under debate, though continues to be reported as a surrogate endpoint for measuring disease progression by international consensus guidelines (20). Second, all patients on UDCA did have a wash out period of 4 weeks and it is possible this was not long enough. Additionally, this population was small and heterogenous, notably that one subject had small duct PSC, which may have a different natural history then large duct PSC. We only used a single donor for all 10 patients in this trial. It is unclear if the results seen could have been obtained with another donor's material. Lastly, it is unclear what the preferred delivery location of FMT material for PSC given that upper GI administration might have more direct effect on the biliary tree microbiota.

Given our design limitations, we would recommend that future studies should consider including GGT, serum bile acids, FGF19 and C4 to assess bile acid synthesis rates and cholestasis as well as serum markers of fibrosis such as ELF and elastography. Although fecal calprotectin was not done, and histology was not collected, a colonoscopy was conducted at baseline and all patients had no endoscopic disease activity.

In conclusion, this is the first human trial of FMT for the treatment of PSC. Here, we demonstrate that FMT is safe in this population. We also noted an improvement in overall microbial diversity that persists after FMT. In addition, we have demonstrated that the abundance of engrafter OTUs present in patients post FMT correlated with a decrease in ALP. Further studies are needed on the utility of isolating those engrafter OTUs as well as 
more broadly to understand the role of the microbiome in the pathophysiology of PSC to permit a significant impact through FMT or more targeted therapy.

\section{Acknowledgments:}

The authors would like to acknowledge PSC Partners for funding this work. 
Table 1: Patient Characteristics

\begin{tabular}{|ll|}
\hline \multicolumn{1}{|l|}{$\mathbf{N}=\mathbf{1 0}$} \\
\hline Male $\mathrm{n}(\%)$ & $8(80 \%)$ \\
\hline Age (mean $\pm \mathrm{SD})$ & $41.1 \pm 15.7$ \\
\hline $\mathrm{UC} \mathrm{n}(\%)$ & $9(90 \%)$ \\
\hline Baseline ALP (mean $\pm \mathrm{SD})$ & $489 \mathrm{U} / \mathrm{L} \pm 274.9$ \\
\hline Baseline ALT (mean $\pm \mathrm{SD})$ & $159.2 \mathrm{U} / \mathrm{L} \pm 62.3$ \\
\hline Baseline AST (mean $\pm \mathrm{SD})$ & $114.4 \mathrm{U} / \mathrm{L} \pm 66.9$ \\
\hline Large Duct PSC n(\%) & $9(90 \%)$ \\
\hline Duration of PSC (mean $\pm \mathrm{SD})$ & $7 \pm 7.1$ years \\
\hline Mayo Risk Score (mean $\pm \mathrm{SD})$ & $-0.007 \pm 0.47$ \\
\hline History of Cholecystectomy & $2(20 \%)$ \\
\hline Baseline Meds & \\
\hline Azathioprine & $6(60 \%)$ \\
\hline Mesalamine & \\
\hline
\end{tabular}




\section{References}

1. Tsaitas C, Semertzidou A, Sinakos E. Update on inflammatory bowel disease in patients with primary sclerosing cholangitis. World journal of hepatology 2014;6:178187.

2. Lazaridis KN, LaRusso NF. Primary Sclerosing Cholangitis. The New England journal of medicine 2016;375:1161-1170.

3. Ricciuto A, Kamath BM, Griffiths AM. The IBD and PSC Phenotypes of PSCIBD. Curr Gastroenterol Rep 2018;20:16.

4. Sinakos E, Marschall HU, Kowdley KV, et al. Bile acid changes after high-dose ursodeoxycholic acid treatment in primary sclerosing cholangitis: Relation to disease progression. Hepatology (Baltimore, Md.) 2010;52:197-203.

5. Tabibian JH, O'Hara SP, Lindor KD. Primary sclerosing cholangitis and the microbiota: current knowledge and perspectives on etiopathogenesis and emerging therapies. Scandinavian Journal of Gastroenterology 2014;49:901-908.

6. Tabibian JH, Talwalkar JA, Lindor KD. Role of the microbiota and antibiotics in primary sclerosing cholangitis. BioMed research international 2013;2013:389537.

7. Tripathi A, Debelius J, Brenner DA, et al. The gut-liver axis and the intersection with the microbiome. Nat Rev Gastroenterol Hepatol 2018;15:397-411.

8. Bjornsson ES, Kilander AF, Olsson RG. Bile duct bacterial isolates in primary sclerosing cholangitis and certain other forms of cholestasis--a study of bile cultures from ERCP. Hepato-gastroenterology 2000;47:1504-1508.

9. Sabino J, Vieira-Silva S, Machiels K, et al. Primary sclerosing cholangitis is characterised by intestinal dysbiosis independent from IBD. Gut 2016;65:1681-1689.

10. Olesen SW, Alm EJ. Dysbiosis is not an answer. Nat Microbiol 2016;1:16228.

11. Farkkila M, Karvonen AL, Nurmi H, et al. Metronidazole and ursodeoxycholic acid for primary sclerosing cholangitis: a randomized placebo-controlled trial. Hepatology (Baltimore, Md.) 2004;40:1379-1386.

12. Tabibian JH, Weeding E, Jorgensen RA, et al. Randomised clinical trial: vancomycin or metronidazole in patients with primary sclerosing cholangitis - a pilot study. Alimentary Pharmacology \& Therapeutics 2013;37:604-612.

13. Cox KL, Cox KM. Oral vancomycin: treatment of primary sclerosing cholangitis in children with inflammatory bowel disease. Journal of pediatric gastroenterology and nutrition 1998;27:580-583.

14. Davies YK, Cox KM, Abdullah BA, et al. Long-term treatment of primary sclerosing cholangitis in children with oral vancomycin: an immunomodulating antibiotic. Journal of pediatric gastroenterology and nutrition 2008;47:61-67.

15. Damman JL, Rodriguez EA, Ali AH, et al. Review article: the evidence that vancomycin is a therapeutic option for primary sclerosing cholangitis. Aliment Pharmacol Ther 2018;47:886-895.

16. Kassam Z, Lee CH, Hunt RH. Review of the emerging treatment of Clostridium difficile infection with fecal microbiota transplantation and insights into future challenges. Clinics in laboratory medicine 2014;34:787-798. 
17. Allegretti J, Eysenbach LM, El-Nachef N, et al. The Current Landscape and Lessons from Fecal Microbiota Transplantation for Inflammatory Bowel Disease: Past, Present, and Future. Inflamm Bowel Dis 2017;23:1710-1717.

18. Chung BK, Hirschfield GM. Immunogenetics in primary sclerosing cholangitis. Curr Opin Gastroenterol 2017;33:93-98.

19. Brunstein CG, Miller JS, Cao Q, et al. Infusion of ex vivo expanded T regulatory cells in adults transplanted with umbilical cord blood: safety profile and detection kinetics. Blood 2011;117:1061-1070.

20. Ponsioen CY, Chapman RW, Chazouilleres O, et al. Surrogate endpoints for clinical trials in primary sclerosing cholangitis: Review and results from an International PSC Study Group consensus process. Hepatology 2016;63:1357-67.

21. Smith MB, Kassam Z, Burgess J, et al. The International Public Stool Bank: A Scalable Model for Standardized Screening and Processing of Donor Stool for Fecal Microbiota Transplantation. Gastroenterology 2015;148 (4):S-211.

22. Dubois N, Ling K, Osman M, et al. Prospective Assessment of Donor Eligibility for Fecal Microbiota Transplantation at a Public Stool Bank: Results From the Evaluation of 1387 Candidate Donors. Open Forum Infect Diseases 2016;2:(suppl 1), 962.

23. Kozich JJ, Westcott SL, Baxter NT, et al. Development of a dual-index sequencing strategy and curation pipeline for analyzing amplicon sequence data on the MiSeq Illumina sequencing platform. Applied and Environmental Microbiology 2013;79:5112-5120.

24. Kummen M, Holm K, Anmarkrud JA, et al. The gut microbial profile in patients with primary sclerosing cholangitis is distinct from patients with ulcerative colitis without biliary disease and healthy controls. Gut 2016.

25. Bajer L, Kverka M, Kostovcik M, et al. Distinct gut microbiota profiles in patients with primary sclerosing cholangitis and ulcerative colitis. World J Gastroenterol 2017;23:4548-4558.

26. Moayyedi P, Surette MG, Kim PT, et al. Fecal Microbiota Transplantation Induces Remission in Patients With Active Ulcerative Colitis in a Randomized Controlled Trial. Gastroenterology 2015;149:102-109.e6.

27. Mullish BH, Pechlivanis A, Barker GF, et al. Functional microbiomics: Evaluation of gut microbiota-bile acid metabolism interactions in health and disease. Methods 2018.

28. Sarafian MH, Lewis MR, Pechlivanis A, et al. Bile acid profiling and quantification in biofluids using ultra-performance liquid chromatography tandem mass spectrometry. Anal Chem 2015;87:9662-70.

29. Sangster T, Major H, Plumb R, et al. A pragmatic and readily implemented quality control strategy for HPLC-MS and GC-MS-based metabonomic analysis. Analyst 2006;131:1075-8.

30. Veselkov KA, Vingara LK, Masson P, et al. Optimized preprocessing of ultraperformance liquid chromatography/mass spectrometry urinary metabolic profiles for improved information recovery. Anal Chem 2011;83:5864-72.

31. Kummen M, Holm K, Anmarkrud JA, et al. The gut microbial profile in patients with primary sclerosing cholangitis is distinct from patients with ulcerative colitis without biliary disease and healthy controls. Gut 2017;66:611-619. 
32. Vital M, Karch A, Pieper DH. Colonic Butyrate-Producing Communities in Humans: an Overview Using Omics Data. mSystems 2017;2.

33. Quraishi MN, Sergeant M, Kay G, et al. The gut-adherent microbiota of PSC-IBD is distinct to that of IBD. Gut 2017;66:386-388.

34. Paramsothy S, Kamm MA, Kaakoush NO, et al. Multidonor intensive faecal microbiota transplantation for active ulcerative colitis: a randomised placebo-controlled trial. Lancet (London, England) 2017.

35. Sitkin S, Vakhitov T, Pokrotnieks J. How to Increase the Butyrate-producing Capacity of the Gut Microbiome: Do IBD Patients Really Need Butyrate Replacement and Butyrogenic Therapy? J Crohns Colitis 2018;12:881-882.

36. Hov JR, Kummen M. Intestinal microbiota in primary sclerosing cholangitis. Curr Opin Gastroenterol 2017;33:85-92. 